\title{
A Comparative Study of Intermaxillary Fixation and Manual Reduction at Open Reduction and Internal Fixation of Mandibular Fractures
}

\author{
Soo-Young Jin, Su-Gwan Kim, Ji-Su Oh, Jeong-Sun Kim ${ }^{1}$, Mi-Ae Jeong ${ }^{2}$ \\ Department of Oral and Maxillofacial Surgery, School of Dentistry, Chosun University, \\ ${ }^{1}$ Department of Dental Hygiene, Gwangju Health University, ${ }^{2}$ Department of Dental Hygiene, Kangwon National University
}

\begin{abstract}
Purpose: The purpose of this study is to evaluate the difference between open reduction and internal fixation (ORIF) and intermaxillary fixation (IMF) of mandibular fractures.

Methods: A total of one hundred twenty-seven patients who were treated for mandibular fractures at Chosun University Dental Hospital, from January 2008 to December 2010, and analyzed their prognoses based on the use of IMF at the time of fracture reduction. The patients were divided into two groups; the manual reduction group without IMF and IMF group. Results: After reduction of the mandibular fracture, good results were obtained with majority patients. Nonetheless, seven patients (13.0\%) in manual reduction method without arch bars or IMF, developed complications after surgery. Three patients underwent IMF due to occlusal instability after surgery, while one patient underwent re-operation. Thus, a significant difference was not observed between the IMF and manual reduction groups.

Conclusion: Manual reduction and IMF at mandibular simple fracture could produce good results. In case of mandibular simple fracture, it was recommended with only manual reduction without IMF or IMF during a short period.
\end{abstract}

Key words: Complication, Fracture, Internal fixation, Mandible

\section{Introduction}

As the society becomes more complex, with increasing population, traffic volume, urbanization, and industrialization, concurrent increases intraffic accidents, industrial accidents, falls, violence, and explosions can be expected. Consequently, craniofacial bone fractures are also expected to increase. Due to the mandible's anatomical protrusion shape, which is unique among facial bones, its fracture incidence is the second highest, after nasal bone fractures[1]. Ugboko et al.[2] have reported that mandibular fractures had $64 \%$ of patients who were treated for maxillofacial fractures. The mandible plays an important role in sensation, occlusion and, mastication. In addition, it determines the appearance of the lower face. Thus, in mandibular fracture cases, accurate reduction is required to recover the facial shape as well as to minimize functional impairments. Not only functional aspects but also esthetic

RECEIVED April 25, 2012, REVISED August 14, 2012, ACCEPTED January 25, 2013

Correspondence to Su-Gwan Kim

Department of Oral and Maxillofacial Surgery, School of Dentistry, Chosun University

309 Pilmun-daero, Dong-gu, Gwangju 501-759, Korea

Tel: 82-62-220-3819, Fax: 82-62-228-7316, E-mail: sgckim@chosun.ac.kr

(c) This is an open access article distributed under the terms of the Creative Commons Attribution Non-Commercial License (http://creativecommons. org/licenses/ by-nc/3.0) which permits unrestricted non-commercial use, distribution, and reproduction in any medium, provided the original work is properly cited. 
elements should be considered.

In the past, many oral and maxillofacial surgeons have primarily used interosseous wiring and intermaxillary fixation (IMF) as preservation treatment methods for the mandibular symphysis. Subsequently, rigid internal fixation was introduced by Michelet et al.[3], which was later modified by Champy et al.[4] and Cawood [5] and has been in widespread use. With the advent of monocortical miniplates, both the occlusion of patients and accurate mandibular reduction have become feasible, raising questions over the necessity of IMF.

This study evaluated 127 patients who were treated for mandibular fractures at Chosun University Dental Hospital from January 2008 to December 2010 and analyzed their prognoses based on the use of IMF at the time of fracture reduction.

\section{Materials and Methods}

A retrospective study was conducted on patients who visited our hospital for a mandibular fracture from January 2008 to December 2010. The cases of midfacial bone fracture, mandibular condyle facture, mixed dentition, and edentulous states were excluded and simple fracture at mandiblular midline, sub-midline, body, and angle was investigated retrospectively (Table 1 ).

The age, gender, and the cause of fracture were examined for each patient. Regarding postsurgical complications, infection, wound dehiscence, osteomyelitis, and malocclusion were evaluated. Panoramic radiographs taken

Table 1. Comparative data

\begin{tabular}{|c|c|c|}
\hline Parameter & Group 1 & Group 2 \\
\hline Number of patients & 73 & 54 \\
\hline Gender & 61 male (83.5\%) & 40 male $(74.1 \%)$ \\
\hline Mean age & 37.3 & 35.2 \\
\hline Age range & $18 \sim 58$ & $20 \sim 64$ \\
\hline \multicolumn{3}{|l|}{ Mechanism of Fx. } \\
\hline Assault & 35 (47.9\%) & 39 (72.2\%) \\
\hline TA & $10(13.7 \%)$ & 4 (7.4\%) \\
\hline Slip down & 17 (23.3\%) & 7 (12.9\%) \\
\hline Fall & $3(4.1 \%)$ & 1 (1.9\%) \\
\hline Sports & $8(10.9 \%)$ & $3(5.6 \%)$ \\
\hline \multicolumn{3}{|l|}{ Fracture site } \\
\hline Symphysis & 7 (9.6\%) & $3(5.6 \%)$ \\
\hline Parasymphysis & $26(35.6 \%)$ & $21(38.9 \%)$ \\
\hline Body & $6(8.2 \%)$ & $2(3.7 \%)$ \\
\hline Angle & 34 (46.6\%) & 27 (50\%) \\
\hline
\end{tabular}

Fx., fracture; TA, traffic accident. immediately after surgery and 1 month after surgery, the loosening of fixation screws, miniplate fractures, and malunion/nonunion were examined. These findings were classified according the severity of complications as follows:

Score 1: patients who could recover with regular sterilization, antibiotic therapy, and simple procedures under local anesthesia

Score 2: patients who required postsurgical IMF or traction using elastic rings because of malocclusion

Score 3: patients who required a second surgery because of osteomyelitis or malunion/non-union

Patients were classified into two groups depending on whether they received IMF. Group 1 included cases where mandibular fracture reduction was achieved using arch bars. Group 2 included cases where manual reduction methods were performed without arch bars or IMF. After the reduction of bone fragments using $2.0 \mathrm{~mm}$ titanium miniplates and monocortical screws, invasive reduction was performed according to the Champy techniques and continuous follow-up visits were conducted. We statistically evaluated inter-group complication ratio, re-operation ratio, and complication score using independent t-test by SPSS version 22.0 (SPSS Inc., Chicago, IL, USA), we think it is statistically significantly important if $P<0.05$.

\section{Results}

Of the 127 patients who satisfied the inclusion and exclusion criteria, IMF was performed on 73 patients (57.5\%), while manual reduction without IMF was performed on 54 patients (42.5\%). In Group 1, 40 patients were male $(83.5 \%)$ while 12 were female (16.5\%). The ages ranged from 18 years to 58 years, with a mean age of 37.3 years. In Group 2, 40 patients were male (74.1\%), while 14 were female (25.9\%). The ages ranged from 20 years to 64 years, with a mean age of 35.2 years. Regarding the cause of fracture, assault was the most prevalent, with 74 patients (58.2\%), followed by slip down in 24 patients (18.9\%), traffic accidents in 14 patients (11.0\%), sports in 11 patients $(8.7 \%)$, and falls in 4 patients $(3.1 \%)$. When they were classified according to the fracture site, the incidence was highest for the mandibular angle (61 patients), followed by the parasymphysis ( 47 patients), symphysis (10 patients), and body (8 patients) (Table 1$)$. 
Table 2. Patient distribution according to complication

\begin{tabular}{lcl}
\hline & Group 1 & Group 2 \\
\hline Normal result $^{\mathrm{a}}$ & $62(84.9 \%)$ & $47(87.0)$ \\
Infection & $4(5.5 \%)$ & $3(5.6)$ \\
Wound dehiscence & $2(2.7 \%)$ & $1(1.9)$ \\
Loosed screw/plate fracture & $2(2.7 \%)$ & 0 \\
Osteomyelitis & $1(1.14 \%)$ & $1(1.9)$ \\
Malocclusion $_{\text {Malunion/nonunion }}$ & $1(1.4 \%)$ & $2(3.7)$ \\
Mean score $^{\mathrm{a}}$ & $1(1.4 \%)$ & 0 \\
Re-operation $^{\mathrm{a}}$ & 1.27 & 1.28 \\
& $1(1.4 \%)$ & 0
\end{tabular}

${ }^{a}$ Evaluation factor, statistical significance is not between two groups $(P>0.05)$.

After reduction of mandibular fractures, good results were obtained in most patients. Nonetheless, after surgery, 7 patients in Group 2 (13.0\%) developed complications. Infection was present in 3 patients, wound dehiscence in 1 patient, and osteomyelitis in 1 patient. The loosening of fixation screws and/or fracture of fixation plates was not observed. Table 2 shows the comparison between Group 1. In 3 patients who presented with malocclusion, normal occlusion was recovered by the application of arch bars and subsequent elastic rings. In one case in Group 1, occlusion failed due to osteomyelitis, and a second surgery was performed under general anesthesia. The average scores for the severity of complications for Group 1 and Group 2 were 1.27 and 1.28, respectively, and a significant difference was not shown.

\section{Discussion}

To date, in the field of oral and maxillofacial surgery, IMF has been performed for the recovery of accurate occlusion and fracture reduction in the treatment of patients with mandibular fractures. IMF is performed by the application of wires, screws for IMF, and arch bars. In particular, arch bars are useful for the re-adjustment of comminuted bone fragments and immobilization of the entire mandible. In addition, arch bars themselves act as tension bands and thus prevent the dispersion of force at the time of reduction. Additionally, in cases involving the failure of initial fixation after surgery or development of slight malocclusion, arch bars can facilitate the attainment of normal occlusion when coupled with elastic rings or wires. In our study, for the 3 patients who developed malocclusion after surgery, arch bars were applied, IMF was performed using elastic rings, and good results were obtained.

However, IMF may cause various side effects. Regarding the use of arch bars, Thor and Andersson[6] have reported that they may injure teeth, adjacent periodontal tissues and the buccal mucosa. Gaujac et al.[7] have reported the possibility of infections due to difficult management and poor oral hygiene, risks for dentists involving skin injuries while using wires and the long treatment times required for the installation and removal of arch bars. In addition, Williams and Cawood[8] have reported that respiratory volume per respiration was decreased by as much as 40\% in patients who underwent IMF. Screws for IMF, however, can reduce opportunistic infections caused by skin injuries and simplify oral hygiene for patients, while the procedure can also be performed more simply over shorter durations. Nonetheless, screws also have the risk of loosening, and screw fractures can lead to tooth injury, among other complications. In patients with multiple comminuted fractures, inedentulous patients, or in patients with a mixed dentition, the use of screws is limited[9]. Coletti et al.[10] have reported that in patients who were treated with miniscrews, 39\% developed side effects. The most prevalent side effect was the loosening of screws for IMF, which was observed $29 \%$ of the time.

In Group 2 of our study, the overall incidence of complications was $13.0 \%$. Compared to Group 1, no statistically significant difference was observed. Regarding cases where a second surgery was performed for serious complications, only 1 case in Group 1 required re-surgery; as such, we could not assess statistical significance compared to Group 2. In addition, when the score was classified according to the seriousness of complications and compared between the two groups, no differences were observed. Bell and Wilson[11] have reported that in the treatment of patients with mandibular angle fractures, complications involving arch bars or Stout wires were not significantly different when compared to complications involving manual reduction. Total of $16 \%$ patients developed complications that required secondary treatments. Nonetheless, most cases were resolved by a simple incision, drainage, and the removal of miniplates. Fordyce et al.[12] have compared occlusal outcomes between a group with IMF and a group without IMF. In the initial period, occlusion was unstable in the group that received manual reduction. After 3 months, 
however, significant differences were not observed. It was considered, however, all three patients occurred malocclusion at this study have had mandibular angle fracture and reduction of mandibular angle fracture through manual reduction should be more carefully decided.

Dimitroulis[13] have reported that manual reduction without IMF has advantages in that it could readily provide a direct visual field for the clinician in comparison with cases involving IMF. For reduction, they recommended that the fixation of miniplates in the proximal bone fragment be performed first, followed by reduction of bone fragments fitting the occlusion, and the fixation of distal bone fragments. Bell and Wilson[11] have reported that cases where the surgery is performed by dentists and inexperienced assistants are not an indication for manual reduction, as IMF is nevertheless required. To obtain accurate occlusion, the roles of aspiration and traction, as well as assistants who have experience in maintaining reduced bone fragments, are all important. In addition, Laurentjoye et al.[14] have reported that in the reduction of bone fragments, accurate reduction of the displaced bone fragments is important if IMF is not performed and that proper anatomical reduction could be obtained by the accurate alignment of the mandibular margins and the conjugation of bone fragments. Dimitroulis[13] also reported that in cases involving manual reduction without IMF, in comparison with cases involving IMF, surgery time could be shortened and early discharge was feasible, thereby reducing treatment costs.

\section{Conclusion}

One hundred twenty-seven patients who were treated for mandibular fractures at Oral and Maxillofacial Surgery of Chosun University Dental Hospital from January 2008 to December 2010 analyzed their prognoses according to IMF at the time of fracture reduction. Among treated patients using manual reduction, malocclusion was occurred in 3 patients, re-operation was required in 1 patient. We could not assess statistical significance compared to the group conducted with IMF. Such mandibular simple fracture could produce good result with even only manual reduction without IMF. In case of mandibular simple fracture, it was recommended only manual reduction without IMF or IMF during short period.

\section{References}

1. Turvey TA. Midfacial fractures: a retrospective analysis of 593 cases. J Oral Surg 1977;35:887-91.

2. Ugboko VI, Oginni FO, Owotade FJ. An investigation into the relationship between mandibular third molars and angle fractures in Nigerians. Br J Oral Maxillofac Surg 2000;38:427-9.

3. Michelet FX, Deymes J, Dessus B. Osteosynthesis with miniaturized screwed plates in maxillo-facial surgery. J Maxillofac Surg 1973;1:79-84.

4. Champy M, Loddé JP, Schmitt R, Jaeger JH, Muster D. Mandibular osteosynthesis by miniature screwed plates via a buccal approach. J Maxillofac Surg 1978;6:14-21.

5. Cawood JI. Small plate osteosynthesis of mandibular fractures. Br J Oral Maxillofac Surg 1985;23:77-91.

6. Thor A, Andersson L. Interdental wiring in jaw fractures: effects on teeth and surrounding tissues after a one-year follow-up. Br J Oral Maxillofac Surg 2001;39:398-401.

7. Gaujac C, Ceccheti MM, Yonezaki F, Garcia IR Jr, Peres MP. Comparative analysis of 2 techniques of double-gloving protection during arch bar placement for intermaxillary fixation. J Oral Maxillofac Surg 2007;65:1922-5.

8. Williams JG, Cawood JI. Effect of intermaxillary fixation on pulmonary function. Int J Oral Maxillofac Surg 1990;19:76-8.

9. Gordon KF, Reed JM, Anand VK. Results of intraoral cortical bone screw fixation technique for mandibular fractures. Otolaryngol Head Neck Surg 1995;113:248-52.

10. Coletti DP, Salama A, Caccamese JF Jr. Application of intermaxillary fixation screws in maxillofacial trauma. J Oral Maxillofac Surg 2007;65:1746-50.

11. Bell RB, Wilson DM. Is the use of arch bars or interdental wire fixation necessary for successful outcomes in the open reduction and internal fixation of mandibular angle fractures? J Oral Maxillofac Surg 2008;66:2116-22.

12. Fordyce AM, Lalani Z, Songra AK, Hildreth AJ, Carton AT, Hawkesford JE. Intermaxillary fixation is not usually necessary to reduce mandibular fractures. $\mathrm{Br} \mathrm{J}$ Oral Maxillofac Surg 1999;37:52-7.

13. Dimitroulis G. Management of fractured mandibles without the use of intermaxillary wire fixation. J Oral Maxillofac Surg 2002;60:1435-8.

14. Laurentjoye M, Majoufre-Lefebvre C, Caix P, Siberchicot F, Ricard AS. Treatment of mandibular fractures with Michelet technique: manual fracture reduction without arch bars. J Oral Maxillofac Surg 2009;67:2374-9. 